\title{
Expression-based decision tree model reveals distinct microRNA expression pattern in pediatric neuronal and mixed neuronal-glial tumors
}

Magdalena Zakrzewska ${ }^{1 *}$ (D), Renata Gruszka', Konrad Stawiski ${ }^{2}$, Wojciech Fendler ${ }^{2,3}$, Joanna Kordacka', Wiesława Grajkowska ${ }^{4,5}$, Paweł Daszkiewicz ${ }^{6}$, Paweł P. Liberski ${ }^{1}$ and Krzysztof Zakrzewski ${ }^{7}$

\begin{abstract}
Background: The understanding of the molecular biology of pediatric neuronal and mixed neuronal-glial brain tumors is still insufficient due to low frequency and heterogeneity of those lesions which comprise several subtypes presenting neuronal and/or neuronal-glial differentiation. Important is that the most frequent ganglioglioma (GG) and dysembryoplastic neuroepithelial tumor (DNET) showed limited number of detectable molecular alterations. In such cases analyses of additional genomic mechanisms seem to be the most promising. The aim of the study was to evaluate microRNA (miRNA) profiles in GGs, DNETs and pilocytic asytrocytomas (PA) and test the hypothesis of plausible miRNA connection with histopathological subtypes of particular pediatric glial and mixed glioneronal tumors.
\end{abstract}

Methods: The study was designed as the two-stage analysis. Microarray testing was performed with the use of the miRCURY LNA microRNA Array technology in 51 cases. Validation set comprised 107 samples used during confirmation of the profiling results by qPCR bioinformatic analysis.

Results: Microarray data was compared between the groups using an analysis of variance with the BenjaminiHochberg procedure used to estimate false discovery rates. After filtration 782 miRNAs were eligible for further analysis. Based on the results of $10 \times 10$-fold cross-validation $J 48$ algorithm was identified as the most resilient to overfitting. Pairwise comparison showed the DNETs to be the most divergent with the largest number of miRNAs differing from either of the two comparative groups. Validation of array analysis was performed for miRNAs used in the classification model: miR-155-5p, miR-4754, miR-4530, miR-628-3p, let-7b-3p, miR-4758-3p, miRPlus-A1086 and miR-891a-5p. Model developed on their expression measured by qPCR showed weighted AUC of 0.97 ( $95 \% \mathrm{Cl}$ for all classes ranging from 0.91 to 1.00). A computational analysis was used to identify mRNA targets for final set of selected miRNAs using miRWalk database. Among genomic targets of selected molecules ZBTB20, LCOR, PFKFB2, SYNJ2BP and TPD52 genes were noted.

Conclusions: Our data showed the existence of miRNAs which expression is specific for different histological types of tumors. miRNA expression analysis may be useful in in-depth molecular diagnostic process of the tumors and could elucidate their origins and molecular background.

Keywords: Brain tumor, Differentiation model, Dysembryoplastic neuroepithelial tumor, Expression, Ganglioglioma, microRNA, Neuronal and mixed neuronal-glial tumor, Pediatric, Pilocytic astrocytoma

\footnotetext{
* Correspondence: magdalena.zakrzewska@umed.lodz.pl

'Department of Molecular Pathology and Neuropathology, Medical

University of Lodz, Pomorska 251, 92-216 Lodz, Poland

Full list of author information is available at the end of the article
}

(c) The Author(s). 2019 Open Access This article is distributed under the terms of the Creative Commons Attribution 4.0 International License (http://creativecommons.org/licenses/by/4.0/), which permits unrestricted use, distribution, and reproduction in any medium, provided you give appropriate credit to the original author(s) and the source, provide a link to the Creative Commons license, and indicate if changes were made. The Creative Commons Public Domain Dedication waiver (http://creativecommons.org/publicdomain/zero/1.0/) applies to the data made available in this article, unless otherwise stated. 


\section{Background}

Pediatric neuronal and mixed neuronal-glial tumors represent about $10 \%$ of all tumors of central nervous system and in the large number of cases are characterized by slow growth and favorable outcome [1]. The frequent manifestation of these relatively rare brain lesions is tumor-related epilepsy responsive to neurosurgical treatment. The taxonomy of the glioneuronal tumors evolved immensely during last two decades; the current WHO classification encompasses 13 entities with still the most frequent dysembryoplastic neuroepithelial tumor (DNET) and ganglioglioma (GG). The classification system changes are the effects of advantages both in histopathological and molecular analyses, however, pediatric low grade tumors lack the multiple genomic alterations observed in higher grade lesions [1-3]. In view of emergent data concerning molecular background of brain tumors further modifications cannot be excluded, especially that understanding of the origin and etiology of neuronal and mixed neuronal-glial tumors may evolve [3-5].

Coexistence of glial and glioneuronal compartment within the tumors as well as their possible histological transformation was an impulse to try to elucidate them in our analysis [6-10]. Recent evidences suggest that in brain tumors, similarly as in the other type of cancer, regulatory aspects of genomic machinery could be essential for tumor pathogenesis, progression and outcome. One of such promising factors is microRNA (miRNA) considered to be one of the crucial transcription modifiers associated with the development of cancer. miRNA analyzes could bring useful and valuable data, especially that only a limited number of structural alterations were observed in the neuronal and mixed neuronal-glial tumors [11-13].

Analysis of such molecular events could also be incorporated into the modern multilayered tumor diagnosis, especially that the miRNA expression seems to be more closely correlated with tumor origin and stage than mRNA status [14, 15]. Nonetheless, the significance of miRNAs expression in rare human disorders remains frequently unclear.

The aim of our study was to elucidate the potential relevance of miRNA expression in glial and glioneuronal pediatric brain tumors. According to that, we performed comprehensive two-stage analysis of miRNA expression in a representative group of those relatively rare pediatric brain tumors with enigmatic molecular background.

\section{Methods}

\section{Patients and tumor samples}

This prospective cohort analysis consisted of a total number of 148 children with low-grade tumors (Table 1, Additional file 1: Table S1).
Table 1 The summary of the clinicopathological features of samples analyzed in the study

\begin{tabular}{ll}
\hline Variable & $N=148$ \\
\hline Age (years) & 9 \\
$\quad$ Median (range 0.5-18) & \\
Gender & $52: 48$ \\
$\quad$ Male (\%): Female (\%) & \\
WHO classification & 45 \\
$\quad$ Pilocytic astrocytoma & 46 \\
Dysembryoplastic neuroepithelial tumor & 57 \\
Ganglioglioma & \\
Location & 118 \\
$\quad$ Supratentorial & 30 \\
Infratentorial & \\
Recurrence & 4 \\
Treatment & \\
$\quad$ Surgery & 148 \\
\hline
\end{tabular}

The group included patients with the diagnosis of lowgrade tumor confirmed by histopathology report (DNET, GG, PA), thus surgically treated according to the standard of care guidelines. All patients were under 18 years of age at the time of surgery. Written informed consent was obtained from all examined individuals. Written informed parental consent was obtained for all patients under 16. For children below 16 years of age the consent was given by parents, for older informed consent was obtained from both parents and child, according to the Polish. The protocol of the study was approved by the Bioethical Committee at the Medical University of Lodz (permit No: RNN/63/14/KE).

\section{Isolation of miRNAs, RNA extraction and CDNA synthesis}

Total RNA, including miRNA fraction was extracted from the tumor tissues stabilized in RNAlater and stored at $-80^{\circ} \mathrm{C}$ until further processing. Samples were processed using a miRNeasy Mini Kit (Qiagen $\mathrm{GmbH}$, Hilden, Germany) in accordance with the manufacturer's protocol. Briefly, samples were incubated at room temperature for a period of $5 \mathrm{~min}$ with $700 \mu \mathrm{l}$ of qiazol lysis reagent. After adding $140 \mu \mathrm{l}$ of chloroform, the samples were shaken vigorously for $15 \mathrm{~s}$, incubated at room temperature for $2 \mathrm{~min}$, and centrifuged for $15 \mathrm{~min}$ at $4{ }^{\circ} \mathrm{C}$. The upper aqueous phase, containing RNA was transferred to a new microcentrifuge tube and mixed with 1.5 volume of $100 \%$ ethanol, transferred to a spin column, centrifuged, washed, and eluted in $30 \mu \mathrm{l}$ RNasefree water.

The quality and quantity of the samples were verified by standard electrophoresis and spectrophotometry methods, with an RNA integrity number (RIN) value $\geq 7$ 
and $\geq 3$ as a cut-off value for microarray and qPCR analysis respectively. For conversion of mature miRNA into cDNA the reverse-transcription reaction mix with Universal cDNA Synthesis Kit (Exiqon, Denmark) was prepared. Each cDNA was stored at $-20^{\circ} \mathrm{C}$ until further analysis.

\section{MiRNA array analysis}

The microarray experiment using the Exiqon platform was performed by Exiqon (Denmark). Briefly, $0.75 \mu \mathrm{g}$ of total RNA from samples were labeled using the miRCURY LNA ${ }^{\mathrm{Tm}}$ microRNA Hi-Power Labeling Kit, $\mathrm{Hy}^{\mathrm{Tm}}{ }^{\mathrm{Tm}} /$ $\mathrm{Hy}^{\mathrm{Tm}}$ and hybridized on the miRCURY LNA $^{\mathrm{ma}}$ microRNA Array 7th Gen (Exiqon, Denmark) using a Tecan $\mathrm{HS} 800^{\mathrm{im}}$ hybridization station (Tecan, Austria). After hybridization the microarray slides were scanned using the Agilent G2565BA Microarray Scanner System (Agilent Technologies, Inc., USA) and the image analysis was carried out using the ImaGene 9 (miRCURY LNA ${ }^{\text {mx }}$ microRNA Array Analysis Software, Exiqon, Denmark).

\section{Quantitative PCR validation}

To confirm the microarray results, during validation stage, the quantitative real-time PCR was performed. qPCR analysis was carried out using ExiLENT SYBR Green master mix (Exiqon, Denmark) on CFX96 ${ }^{\text {ma }}$ Touch Real-Time PCR Detection System (Bio-Rad, Germany) with custom selection of miRCURY LNA $^{\text {ta }}$ primers in Pick-\&-Mix, 96 well Ready-to-Use formats. The PCR reactions for each miRNA were run in duplicate and the results were averaged over these analyzes. The normalized relative expression level of the genes of interest was calculated according to the $\mathrm{dCt}$ method.

\section{Data preprocessing and statistical analysis}

All data analysis and interpretation were performed by us. Microarray data normalization was performed to minimize differences between the colors in an intensitydependent manner using Lowess (Locally Weighted Scatterplot Smoothing) regression algorithm. miRNAs measured in less than $80 \%$ of cases were filtered out. Expression data were $\log 2$-transformed and compared using one-way analysis of variance. Expression of all remaining miRNAs was compared between the three groups using an analysis of variance (ANOVA) with the Benjamini-Hochberg procedure used to estimate false discovery rates (FDR). To adjust for multiple hypothesis testing false FDR were calculated for all miRNAs with ones characterized by $p<0.05$ and FDR $<0.15$ being considered as significant for the purpose of classifier development and further validation. Additional post-hoc analysis was performed to further clarify the differential expression between the groups.
To provide further insights into tumors biology, miRNA evaluation of microarray samples was also analyzed in gangliogliomas with BRAFV600E mutation (5/ 19). Differential analysis was performed using unpaired t-test with FDR correction.

Expression of miRNAs in the validation cohort was calculated according to the $\mathrm{dCt}$ and results were again compared between the groups using one-way analysis of variance, followed by Tukey's post-hoc test if significant results $(p<0.05)$ were observed in ANOVA. Hierarchical clustering heatmaps were constructed for both the primary and validation groups using Euclidean distance and complete linkage protocol. Similarly, in the validation cohort, a classification model was developed. The missing expression values in validation cohort prior to data mining modeling were imputed using expectationmaximization algorithm with a multivariate normal model. STATISTICA 13 (Statsoft, Tulsa, OK, USA), MultiExperiment Viewer (Dana-Farber Cancer Institute, Boston, MA, USA) and several R packages (caret, mice, RWeka) were used for statistical analysis [16-18].

Complex data-mining analysis was conducted using Waikato Environment for Knowledge Analysis (WEKA 3.9.0, University of Waikato, New Zeeland). Feature selection based on difference significance in differential expression analysis was performed to decrease dimensionality in microarray data. Subsequently, to evaluate miRNAs subsets using classification model induction were assessed in 10 times 10-fold crossvalidation manner. Six modeling approaches were tested in profiling stage, J48 algorithm, ZeroR algorithm, JRip algorithm, random tree and support vector machine with radial basis function kernel [19]. In validation stage the best performing approach was used to induct model confirming the observations from profiling stage. The details of methods used in the modeling with their hyperparameters were provided as supplementary material (Additional file 2: Table S2).

A computational analysis was used to identify mRNA targets for final set of selected miRNAs using miRWalk database [20].

\section{Results}

The final cohort was comprised of 46 cases of DNET (WHO grade I), 57 cases of GG (WHO grade II) and 45 cases of PA (WHO grade I) classified according to the criteria of WHO updated classification [1]. Fifty-one and 107 samples were included in profiling and in validation stage respectively. All patients were under 18 years of age at the time of surgery. Median age was 9 years (range: 5 months to 18 years). Profiling and validation GG subgroups were independent set of samples. In the DNET and PA subgroups five and six specimens, 
respectively, were collected in both sets (Additional file 1: Table S1).

The array quality assessment showed successful labeling for the control spike-in oligonucleotides in the expected range and normalization of the quantified signals using the global LOWESS regression algorithm was performed. Filtration of expression data of 51 microarray samples showed 782 miRNAs eligible for detailed analysis (Additional file 3: Table S3). Differential expression analysis performed in ANOVA showed 10 miRNAs meeting the prespecified criteria significance in tumor subgroups differentiation (Fig. 1a, Additional file 4: Table S4). Pairwise comparisons of the three analyzed groups: DNET, GG and PA showed the DNET tumors to be the most divergent with the largest number of miRNAs differing from either of the two comparative groups (Fig. 1b).

The results presented as the weighted average of 10 random 10-fold cross-validation of modeling for each feature set indicted the J48 algorithm with significance-

\section{a}
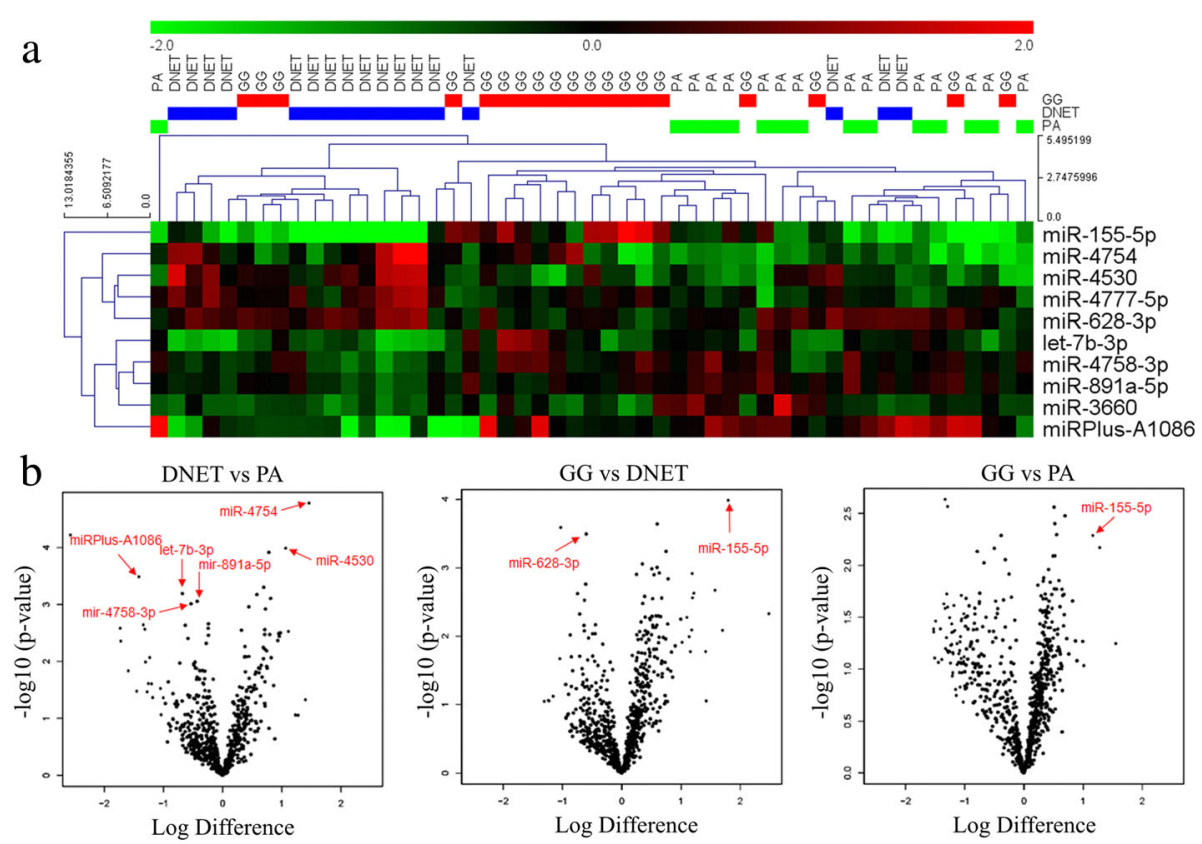

c

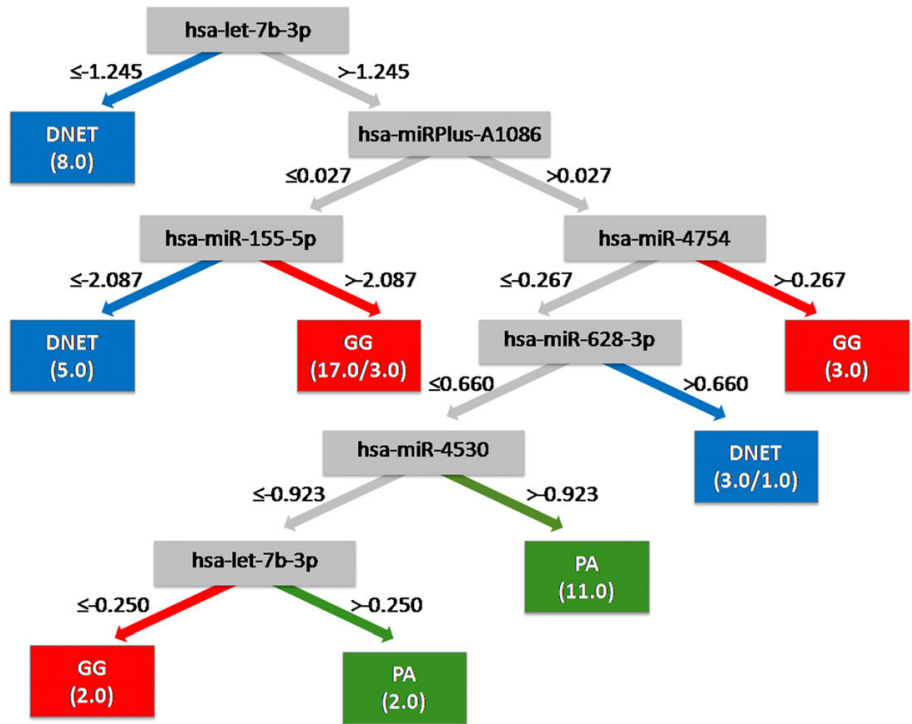

Fig. 1 Results of microarray analysis performed in the profiling stage. a Heatmap of significantly differently expressed miRNAs between the tumor subgroups; $\mathbf{b}$ The volcano plots of all three comparisons with miRNAs chosen for validation stage indicated by their labels; $\mathbf{c}$ The best performing model achieved by application of J48 algorithm on the set of miRNAs selected by significance-based method. DNET dysembryoplastic

neuroepithelial tumor, GG ganglioglioma, PA pilocytic astrocytoma 
Table 2 Weighted area under ROC curve for different feature selection and classifier induction methods showed J48 with significance-based feature selection as the one of the best performing and immune to overfitting modeling approaches. Significance filter was chosen to be preferable based on the highest average across multiple feature selection methods. J48 has been chosen as the best method based on the highest average across all methods

\begin{tabular}{|c|c|c|c|c|c|c|c|}
\hline Dataset & $J 48$ & ZeroR & Jrip & Decision Stump & Random Tree & SVM & Average \\
\hline Full dataset & 0,59 & 0,50 & 0,63 & 0,66 & 0,59 & 0,61 & 0,60 \\
\hline Significant miRNAs & 0,71 & 0,50 & 0,71 & 0,60 & 0,67 & 0,50 & 0,62 \\
\hline Support vector machine-based attribute evaluator & 0,66 & 0,50 & 0,62 & 0,49 & 0,61 & 0,50 & 0,56 \\
\hline TTP & 0,55 & 0,50 & 0,52 & 0,49 & 0,53 & 0,51 & 0,52 \\
\hline Average & 0,63 & 0,50 & 0,62 & 0,56 & 0,60 & 0,53 & \\
\hline
\end{tabular}

SVM Support vector machine with radial basis function kernel, TTP Targeted projection pursuit

based feature selection as the method mostly resilient to overfitting (Table 2).

The final model on microarray data showed accuracy of $92.2 \%$ with weighted area under the ROC curve of 0.971 ( $p<0.01$, Fig. 1c).

Selective miRNA analysis performed for five GGs carrying BRAFV600E mutation presented miRNAs which were significantly differentially expressed and none of those were used for final classification (Additional file 5: Table S5).

Proposed approach has confirmed the potential for a miRNA-based classifier which we attempted to calibrate on a separate validation cohort. Validation samples underwent expression measurement using a pick-andmix array consisting of 8 miRNAs identified using microarray expression profiling and that were crucial for the best model development. Additionally, five normalizers were selected as the most stable reference miRNAs with the NormFinder algorithm [21] (Table 3).

Table 3 miRNAs used for qPCR validation of microarray data

\begin{tabular}{clll}
\hline No & miRNA & Sequence & Assay symbol \\
\hline Differential Expression & & \\
1 & miR-155-5p & UUAAUGCUAAUCGUGAUAGGGGU & 204,308 \\
2 & miR-4754 & AUGCGGACCUGGGUUAGCGGAGU & $2,107,017$ \\
3 & miR-4530 & CCCAGCAGGACGGGAGCG & $2,105,012$ \\
4 & miR-628-3p & UCUAGUAAGAGUGGCAGUCGA & 206,057 \\
5 & let-7b-3p & CUAUACAACCUACUGCCUUCCC & 205,653 \\
6 & miR-4758-3p & UGCCCCACCUGCUGACCACCCUC & $2,118,014$ \\
7 & miR-891a-5p & UGCAACGAACCUGAGCCACUGA & 204,220 \\
8 & miRPlus-A1086 & UAGUGCCGUGGUCCUUUUGGC & 169,416 \\
Stable Expression & & \\
1 & miR-500a-5p & UAAUCCUUGCUACCUGGGUGAGA & 204,794 \\
2 & miR-451b & UAGCAAGAGACCAUUACCAUU & $2,103,713$ \\
3 & miR-514b-3p & AUUGACACCUCUGUGAGUGGA & $2,108,297$ \\
4 & miR-1293 & UGGGUGGUCUGGAGAUUUGUGC & $2,110,424$ \\
5 & miR-1226-3p & UCACCAGCCCUGUGUUCCCUAG & $2,102,736$ \\
\hline
\end{tabular}

Results of the qPCR validation also showed that all three types of analyzed tumors exhibit distinct miRNA expression profiles (Fig. 2, Additional file 6: Table S6). Pairwise comparisons of the miRNAs showed that the differences in miRNA expression in the validation analysis were in line with those observed in the profiling experiment (Additional file 7: Table S7). Significant differences between the groups were identified for miR4754, miR-4530, miR-155-5p, miR-4758-3p and miR$628-3 \mathrm{p}$. These results confirmed the differences noted in the profiling experiment: miR-4754 showed significantly higher expression in the DNET group than in PA; miR4530, miR-155-5p and miR-628-3p had the lowest expression in PA, DNET and GG tumors respectively. However, expression of miR-4758-3p was the highest in the DNET samples, which was not a direct confirmation of the microarray experiment, as this group showed the lowest expression previously. The expression of miR4754 at low level in 55 out of 107 samples rendered it useless for the purpose of developing a classification model.

Classification model developed on the expression of selected miRNAs for qPCR validation showed the accuracy of $90.7 \%$ and weight AUC of 0.97 in validation on training set with possibility of developing an accurate decision tree and was comparable to the results obtained on the profiling stage of analysis (Table 4, Fig. 3).

Performed here in silico prediction of miRNA-mRNA interactions showed $L N P K$ and LCOR targets as targeted by more than one of miRNAs (Additional file 8: Table S8). In case of predicted targets, 6 of 8 selected miRNAs were expected to target ZBTB20, LCOR, PFKFB2, SYNJ2BP and TPD52 genes (Additional file 9: Table S9).

\section{Discussion}

Here we studied the miRNA expression in the pediatric glial and mixed glioneuronal tumors. The comparison of dysembryoplastic neuroepithelial tumor, ganglioglioma and pilocytic astrocytoma gave us the information concerning molecular differences detected on the miRNA level. Bioinformatic analysis revealed a number of 


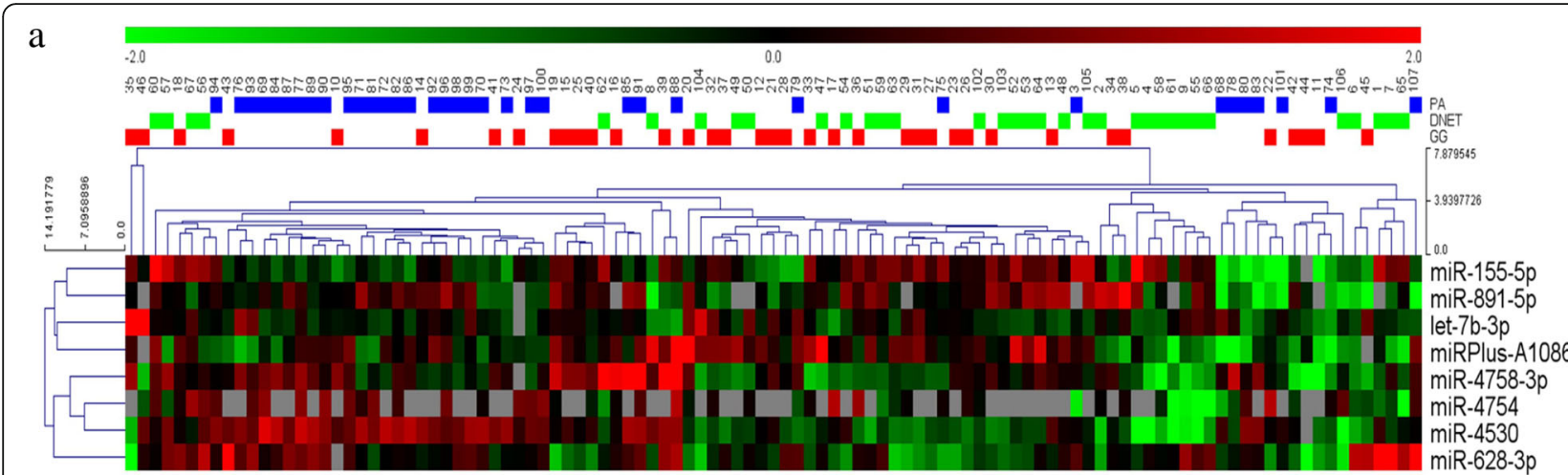

b
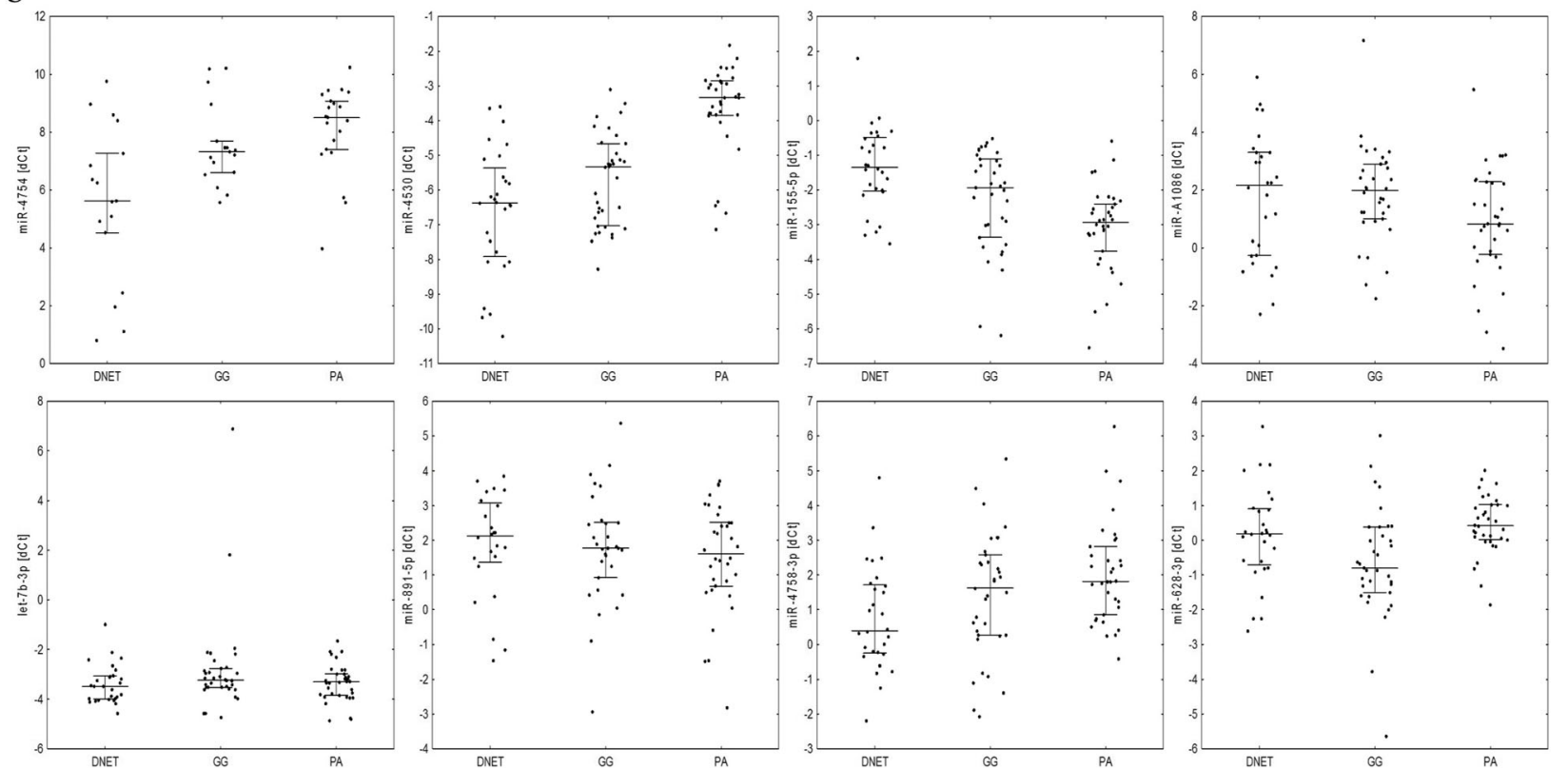

Fig. 2 Results of analysis performed in the validation stage. a Heatmap of the best miRNA qualifiers differentiating between the tumors subgroups validated by using qPCR; $\mathbf{b}$ Dot plot showing the relationship between the expression of selected miRNAs and histopathological type of tumor. DNET dysembryoplastic neuroepithelial tumor, GG ganglioglioma, PA pilocytic astrocytoma

miRNAs differently expressed in each tumor type with the largest number of miRNAs differentiating DNETs from the two comparative groups. Noteworthy, our models could be used to differentiate the type of tumor without comparison to normal tissue. This is because the miRNAs of stable expression were used as comparators, thus their measurement and subsequent use in normalization is required.

At present little is known about significance of miRNA expression in pediatric glial and mixed tumors and there are scanty data concerning detailed expression analysis of such cases. Previously published miRNA profiling studies were performed on small numbers of cases, which frequently mixed adult and pediatric samples $[12$, 22-27] (Table 5). Additionally, the analyses performed so far mainly focused on the differences between normal, peritumoral and tumor tissue [22-24]. Such approaches were most probably a consequence of low frequency of mixed glioneuronal tumors occurring in pediatric population.

We analyzed miRNA profiles of a large number of pediatric samples and confirmed their efficient differentiation on the basis of miRNA expression levels. The decision tree modeling algorithm used by us reached an AUC value 0.97 both in profiling and validation set of samples, which indicates for high discriminatory power of selected miRNAs. These findings indicated that the expression of miRNAs is representative for histopathological subtypes of analyzed tumors of similar origin. Correct classification of glial and glioneuronal pediatric tumors with accuracy of 92.2 and $90.7 \%$ on array and validation stage respectively demonstrated high accuracy of the proposed model by combining the expression levels of 8 miRNAs. 
Table 4 miRNA expression fold changes achieved on profiling and validation set of samples for the three analyzed subgroups

\begin{tabular}{|c|c|c|c|}
\hline miRNA & PA vs. DNET & DNET vs. GG & PA vs. GG \\
\hline \multicolumn{4}{|l|}{ Profiling set FC } \\
\hline hsa-miR-4754 & $0,365,273,252$ & $1,734,884,736$ & $0,633,706,989$ \\
\hline hsa-miR-4530 & $0,486,237,157$ & $1,884,327,942$ & $0,91,623,026$ \\
\hline hsa-miR-155-5p & $1,544,860,921$ & $0,310,106,478$ & $0,479,071,379$ \\
\hline hsa-miRPlus-A1086 & $2,919,325,657$ & $0,563,816,578$ & $1,645,964,203$ \\
\hline hsa-let-7b-3p & $1,670,915,943$ & $0,631,840,741$ & $1,055,752,768$ \\
\hline hsa-miR-891a-5p & 1,364,996,166 & $0,844,233,833$ & $1,152,375,946$ \\
\hline hsa-miR-4758-3p & $1,462,678,698$ & $0,72,361,195$ & $1,058,411,786$ \\
\hline hsa-miR-628-3p & $0,821,392,107$ & $1,471,905,349$ & $1,209,011,436$ \\
\hline \multicolumn{4}{|l|}{ Validation set FC } \\
\hline hsa-miR-4754 & $0,142,744,049$ & $12,14,192,594$ & $1,733,187,674$ \\
\hline hsa-miR-4530 & 0,071030983 & $4,177,155,132$ & $0,296,707,437$ \\
\hline hsa-miR-155-5p & $3,883,969,214$ & $0,358,060,831$ & $1,390,697,245$ \\
\hline hsa-miRPlus-A1086 & $1,544,571,733$ & $1,576,092,261$ & $2,434,387,555$ \\
\hline hsa-let-7b-3p & $0,989,336,539$ & $1,189,024,878$ & $1,176,345,758$ \\
\hline hsa-miR-891a-5p & $240,712,865$ & $0,766,431,411$ & $1,844,899,007$ \\
\hline hsa-miR-4758-3p & 0,392,739,993 & $1,189,309,823$ & $0,467,089,532$ \\
\hline hsa-miR-628-3p & $0,597,175,923$ & $0,418,895,902$ & $0,250,154,547$ \\
\hline
\end{tabular}

DNET Dysembryoplastic neuroepithelial tumor, FC Fold change, GG Ganglioglioma, PA Pilocytic astrocytoma

Although for some of these miRNAs the relationship to brain tumor formation was mostly unknown, among them there were also extensively studied molecules such as highly conserved miR-155 with confirmed altered expression in various types of cancers [25, 28-30]. It has an assigned oncogenic function, also confirmed in gliomas with plausible role in tumor development and progression [31]. Higher levels of miR-155 were observed in tumor specimens and peritumoral tissue in gliomas of different grade and histopathological features [29]. Methylation of gene promoter region reduced the miRNA expression and was correlated with longer survival of patients with glial tumors [30]. It was also considered as a potential molecular treatment target due to the correlation with WNT pathway [31]. On the basis of up-regulation in macrophages, monocytes, and microglia in response to pro-inflammatory stimuli the molecule was also regarded as an important factor involved in inflammatory processes [32, 33].

Our observation of higher expression levels of miR155-5p in DNETs and GGs indicated for its potential role in tumors coupled with neuronal dysfunction. Presence of that miRNA in the proposed expression-based decision tree for differentiation of mixed tumors also strongly indicates for miR-155-5p significance in brain tumor biology. Such observations suggest possibility of new treatment modalities influencing miRNA expression especially in patients with drug-resistant epilepsy and higher levels of miR-155 [25, 34].

Furthermore miR-155-5p was one out of four miRNAs positively validated by us, with a putative relation to developmental and/or inflammatory processes. In that context the value of tumors inflammatory microenvironment seems to be important during the development of glial and mixed neuronal-glial tumors. Up to now such association was underlined by Prabowo et al. in their analysis of three miRNAs involved in the regulation of the pro-inflammatory pathways, miR-146a, miR-21, and miR-155 [25]. The observation concerning relationship between miRNA expression and developmental delay, including intellectual disability and seizures, was noted also by Carvalheira et al. on the basis of molecular analysis of patients with chromosome 19 rearrangements [32]. They indicated that miR-4754, which has also been selected in our study, could be related to brain development mainly on the basis of its possible target genes JMY, CNTN3 and EYA4 [32].

Performed here computational in silico analysis of miRNA-mRNA network also showed connection with genes expressed during human brain development, $L N P K$ and ZBTB20. Two out of 8 selected miRNAs have shown to target $L N P K$, encoding the endoplasmic reticulum junction stabilizer lunapark, gene with assigned role in neurodevelopmental disorders and plausible contribution to the epilepsy [35]. In turn transcriptional repressor $Z B T B 20$ had confirmed function in neural precursor cells [36]. Moreover for ZBTB20 and PFKFB2 genes plausible link between genes alteration and glioma proliferation and invasion was underlined, especially in the context of glioma-associated cells [37, 38]. Similar function was assigned to another gene selected in our analysis, miR-4530 which plausibly contributes to the promotion of tumor angiogenesis in endothelial cells and possibly to tumor malignant transformation [39-41]. Pro-angiogenic function in tumor formation was also reported for oncomiR-891a-5p which has a suspected pro-tumorigenic role [42, 43]. Reports exist for involvement of miR-628-3p, miR-4758-3p, let7b-3p and miRPlus-A1086 in pro-tumoral networks. Up-regulation of miR-628-3p was observed in different types of extracranial tumors in adult and pediatric patients [44]. What is more, the molecule's altered expression was observed in patients with Huntington's disease and it could be interpreted as an indirect evidence for its involvement in brain function and development [45-48]. let-7b has suspected tumor suppressor function in gliomas and low expression of the let-7 family members may be responsible for the poor prognosis of brain tumors patients through disturbed inhibition of KRAS, HMGA2 and $M Y C$ oncogenes [49-51]. Our study indicated for following potential targetable biomarker, TPD52 which 


\section{a}

a
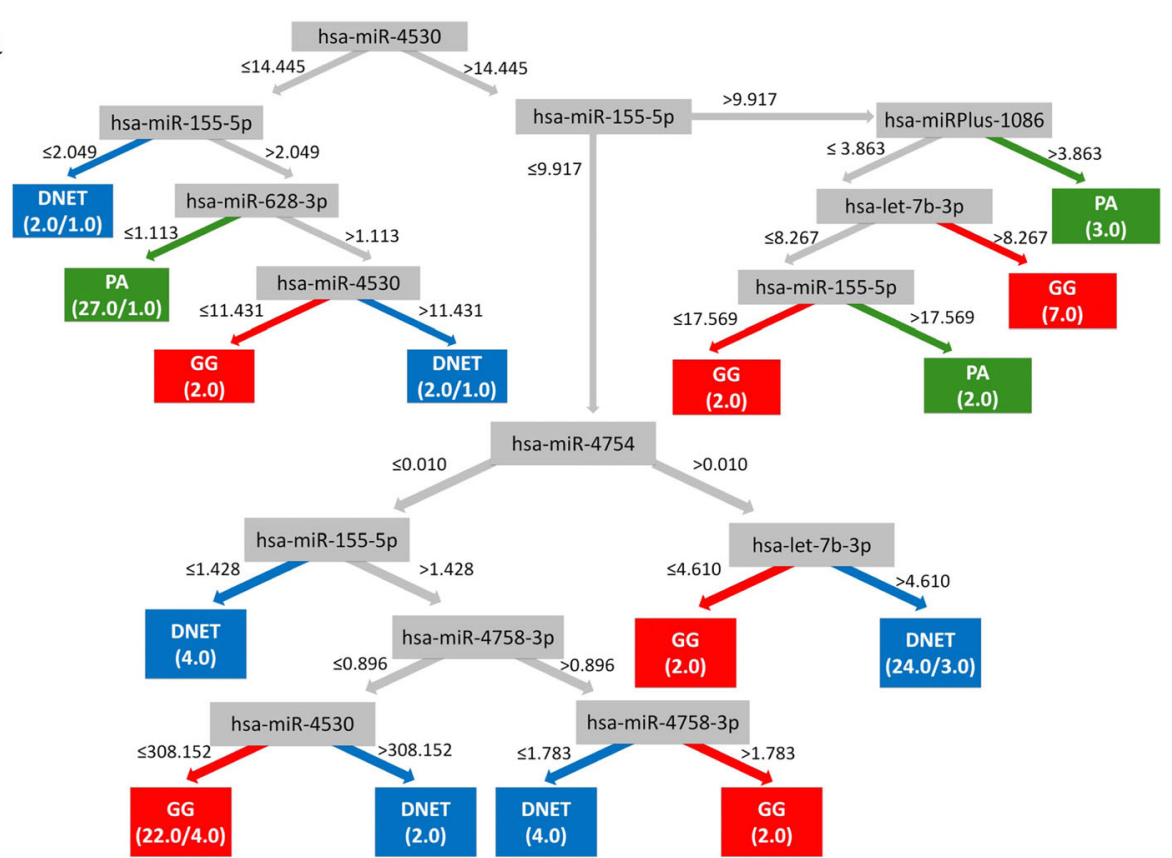

$\mathrm{b}$

DNET ROC CUIve

GG ROC Curve
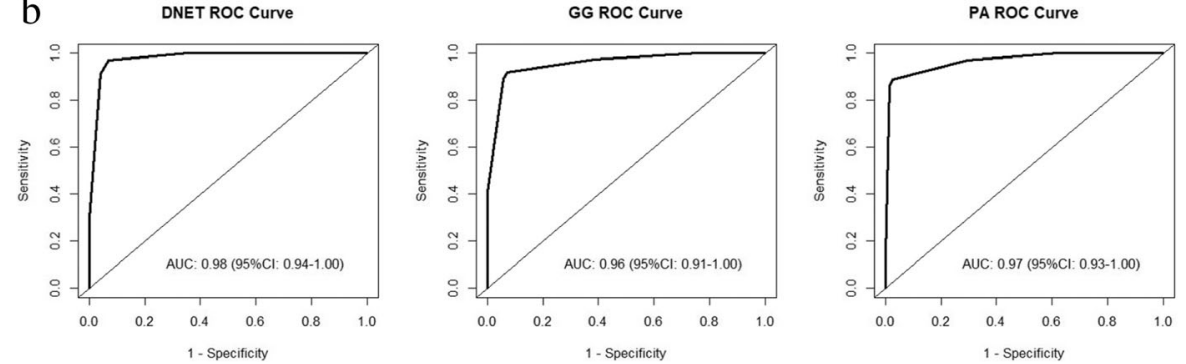

Fig. 3 Final decision tree developed on GPCR validation dataset. a The structure of best performing expression-based decision tree for differentiation of samples; $\mathbf{b}$ The predictive abilities of the model by ROC analysis. Class probability estimates based on observed relative class frequencies at the leaf nodes were used for the development of ROC curves. AUC area under the ROC curve, DNET dysembryoplastic neuroepithelial tumors, GG gangliogliomas, PA pilocytic astrocytomas, ROC receiver operating characteristic curve

Table 5 Review of the literature data concerning microRNA analyses in glioneuronal tumors

\begin{tabular}{|c|c|c|c|c|}
\hline $\begin{array}{l}\text { No of } \\
\text { samples }\end{array}$ & $\begin{array}{l}\text { Tumor } \\
\text { type }\end{array}$ & $\begin{array}{l}\text { Age } \\
\text { category }\end{array}$ & Summary & Ref. \\
\hline 41 & $\begin{array}{l}\text { mixed } \\
\text { LGG }\end{array}$ & C/A & $\begin{array}{l}61 \text { differently expressed miRNAs on profiling study }(n=41) \text {; the same samples }(n=19) \text { used for validation of } \\
\text { miR-129-2-3p, miR-219-5p, miR-338-3p, miR-487b, miR-885-5p, miR-323a-3p, miR-4488 and miR-1246; expres- } \\
\text { sion of miR-487b from cluster } 14 q 32.31 \text { variably underexpressed in pediatric glioma lines }\end{array}$ & [24] \\
\hline 34 & GG & $\mathrm{C} / \mathrm{A}$ & evaluation of inflammation-related miR-21, miR-146, miR-155; miR-146 contributed to epileptogenic network & [25] \\
\hline 29 & GG & C/A & $\begin{array}{l}66 \text { miRNAs differently expressed on profiling study }(n=9) \text {; validation }(n=20) \text { of miR-217 showed relationship } \\
\text { between miR-217 and ERK1/2 }\end{array}$ & [22] \\
\hline 5 & DNET & C & $\begin{array}{l}120 \text { differently expressed miRNAs on profiling study }(n=5) \text {; the same samples used for validation aof down- } \\
\text { regulated miR-3138 and overexpressed miR-1909 }\end{array}$ & [23] \\
\hline 43 & PA & C/YA & 31 differently expressed miRNAs on profiling study $(n=43)$; validation of miR-21, miR-124 and mir-129 $(n=9)$ & [12] \\
\hline 99 & $\begin{array}{l}\text { mixed } \\
\text { LGG }\end{array}$ & C/A & miR-519d and miR-4758 upregulated in GGs compared to control tissue, DNET and other gliomas & [50] \\
\hline
\end{tabular}


overexpression were noted in many cancer types including brain tumors $[52,53]$. Here we showed that in case of predicted targets, 6 of 8 selected miRNAs were expected to target TPD52 gene during in silico prediction.

Ultimately, the limited information concerning miRPlus-A1086 showed their relationship to treatment modalities in prostate cancer and melanoma [52, 54].

Taking the above into consideration, it seems that all the miRNAs selected in the current study have previously been reported as functional in cancers, what suggests that the selection method detected not only the most differentially expressed molecules but also identify biologically important miRNAs $[45-48,55]$.

Recent analysis published by Bongaarts et al. showed miR-4758 as up-regulated in GG compared to control cortex with AUC reaching 0.837. The differences were not significant compared with PA and confirmed for DNETs [56]. Here we revealed the classifiable utility for miR4758-3p together with remaining 7 miRNAs for pediatric neuronal and mixed neuronal-glial brain tumors with high reproducibility of results. This was likely made possible due to the number of genes selected for the proposed model, a large number of samples included in the analysis, as well as limiting the sample pool only to the pediatric population. It enables us to define characteristic miRNA profile of different tumors of neuroepithelial origin with decision tree showing tremendous performance on training set and in leave-one-out cross-validation.

\section{Conclusions}

Here we showed that distinct miRNA expression pattern is a specific feature of different pediatric low grade gliomas. A proposed classification model based on the set of miRNA may be a useful classification tool in cases that are difficult to distinguish by classical histopathological examination. Known biological stability of miRNA indicates for usefulness of proposed decision tree in gangliogliomas, dysembryoplastic neuroepithelial tumors and pilocytic astrocytomas classification by using qPCR method, which is widely recognized as the gold standard for analyzing the expression of miRNAs.

The study has also a potential to increase our understanding of the molecular mechanisms of brain tumors development. Additionally, our observations suggest that histological transformation between glial and mixed pediatric tumors could be a consequence of new alterations occurring during cancerogenesis which lead to morphogenetic changes regardless of core molecular alterations.

\section{Additional files}

Additional file 1: Table S1. Tumor detailed characteristics. * indicates samples used in profiling experiment, ${ }^{*} \wedge$ indicates samples included both in profiling and validation cohort. DNET dysembryoplastic neuroepithelial tumor, GG ganglioglioma, PA pilocytic astrocytoma. (XLSX $16 \mathrm{~kb}$ )

Additional file 2: Table S2. Configuration of hyperparameters set a priori for modelling in WEKA software. (XLSX $9 \mathrm{~kb}$ )

Additional file 3: Table S3. Log2-transformed expression data of samples assessed using microarrays in profiling stage. DNET dysembryoplastic neuroepithelial tumor, GG ganglioglioma, PA pilocytic astrocytoma. (XLSX $1599 \mathrm{~kb})$

Additional file 4: Table S4. Results of microarray data pairwise comparisons between the three groups. AVG average, DNET dysembryoplastic neuroepithelial tumor, FC fold change, FDR false discovery rate, GG ganglioglioma, PA pilocytic astrocytoma, SD standard deviation. (XLSX $182 \mathrm{~kb})$

Additional file 5: Table S5. List of miRNAs differentially expressed in BRAFV600E mutated and wild type cases (GG12, GG25, GG28, GG33, GG55). Negative fold change indicates downregulation in mutated cases. miRNAs selected for validation has been appended to the Table. FC fold change, FDR false detection rate. (XLSX $11 \mathrm{~kb}$ )

Additional file 6: Table S6. Transformed delta $\mathrm{Ct}(2 \wedge-d C t)$ values of miRNAs expression obtained in validation stage. (XLSX $25 \mathrm{~kb}$ )

Additional file 7: Table S7. Results of pairwise comparisons between the three groups in $\mathrm{PPCR}$ validation. AVG average, DNET dysembryoplastic neuroepithelial tumor, $F C$ fold change, FDR false discovery rate, GG ganglioglioma, PA pilocytic astrocytoma, SD standard deviation. (XLSX $11 \mathrm{~kb}$ )

Additional file 8: Table S8. Genes targeted by selected 8 miRNAs along with the list of targeting miRNAs. Only experimentally validated targets have been summarized in this table showing results of in silico analysis. (XLSX $11 \mathrm{~kb}$ )

Additional file 9: Table S9. Genes targeted by selected 8 miRNAs along with the list of targeting miRNAs. Experimentally validated and predicted targets have been summarized in this table showing results of in silico analysis. (XLSX $164 \mathrm{~kb}$ )

\section{Abbreviations}

AUC: Area under the receiver operating characteristic curve; AVG: Average; DNET: Dysembryoplastic neuroepithelial tumor; FC: Fold change; FDR: False discovery rate; GG: Ganglioglioma; miRNA: MicroRNA; PA: Pilocytic astrocytoma; qPCR: Quantitative real-time polymerase chain reaction; ROC: Receiver operating characteristic curve; SD: Standard deviation; SVM: Support vector machine with radial basis function kernel; TTP: Targeted projection pursuit

\section{Acknowledgements}

Not applicable.

\section{Authors' contributions}

Designed and coordinated the study: MZ, RG. Performed the experiments: $M Z, R G$, JK. Performed histopathology examinations: WG, PPL. Analyzed and interpreted the data: MZ, KS, WF, JK, RG. Wrote the paper: MZ, KS. Provided samples for experiments and obtained clinical data: PD, KZ. All authors read and approved the final manuscript.

\section{Funding}

This study was supported by the National Science Center Grant No. 2014/15/ B/NZ4/00744. The funders had no role in the design of the study and collection, analysis, and interpretation of data and in writing the manuscript.

\section{Availability of data and materials}

The datasets used and/or analyzed during the current study are available from the corresponding author on reasonable request.

\section{Ethics approval and consent to participate}

Written informed consent was obtained from all examined individuals. Written informed parental consent was obtained for all patients under 16 . For children below 16 years of age the consent was given by parents, for older informed consent was obtained from both parents and child, according to the Polish law (Act of 6 November 2008 on Patients' Rights and the Patient's Rights Ombudsman, Dz.U.2017.1318, as applicable from 
February 19, 2019). The protocol of the study was approved by the Bioethical Committee at the Medical University of Lodz (permit No: RNN/63/14/KE).

\section{Consent for publication}

Not applicable.

\section{Competing interests}

The authors declare that they have no competing interests.

\section{Author details}

${ }^{1}$ Department of Molecular Pathology and Neuropathology, Medical University of Lodz, Pomorska 251, 92-216 Lodz, Poland. ${ }^{2}$ Department of Biostatistics and Translational Medicine, Medical University of Lodz, Mazowiecka 15, 92-215 Lodz, Poland. 'Department of Radiation Oncology, Dana-Farber Cancer Institute, Boston, MA, USA. ${ }^{4}$ Department of Pathology, The Children's Memorial Health Institute, Av. Dzieci Polskich 20, 04-730 Warsaw, Poland. ${ }^{5}$ Department of Experimental and Clinical Neuropathology, Mossakowski Medical Research Centre, Pawinskiego 5, 02-106 Warsaw, Poland. 'Department of Clinical Department of Neurosurgery, The Children's Memorial Health Institute, Av. Dzieci Polskich 20, 04-730 Warsaw, Poland. ${ }^{7}$ Department of Neurosurgery, Polish Mother Memorial Hospital Research Institute in Lodz, Rzgowska 281/289, 93-338 Lodz, Poland.

\section{Received: 24 October 2018 Accepted: 22 May 2019}

\section{Published online: 06 June 2019}

\section{References}

1. Louis DN, Ohgaki H, Wiestler OD, Cavenee WK, editors. WHO classification of tumors of the central nervous system. 4th ed. Lyon: IARC Press; 2016.

2. Gupta K, Orisme W, Harreld JH, Qaddoumi I, Dalton JD, Punchihewa C, et al. Posterior fossa and spinal gangliogliomas form two distinct clinicopathologic and molecular subgroups. Acta Neuropathol Commun. 2014;:18.

3. Lassaletta A, Zapotocky M, Bouffet E, Hawkins C, Tabori U. An integrative molecular and genomic analysis of pediatric hemispheric low-grade gliomas: an update. Childs Nerv Syst. 2016;32(10):1789-97.

4. Heiland DH, Staszewski O, Hirsch M, Masalha W, Franco P, Grauvogel J, et al. Malignant transformation of a Dysembryoplastic Neuroepithelial tumor (DNET) characterized by genome-wide methylation analysis. J Neuropathol Exp Neurol. 2016;75(4):358-65.

5. Wood MD, Tihan T, Perry A, Chacko G, Turner C, Pu C, et al. Multimodal molecular analysis of astroblastoma enables reclassification of most cases into more specific molecular entities. Brain Pathol. 2018;28(2):192-202.

6. Fiset PO, Fontebasso AM, De Jay N, Gayden T, Nikbakht H, Majewski J, et al. Longitudinal mutational analysis of a cerebellar pilocytic astrocytoma recurring as a ganglioglioma. Pediatr Blood Cancer. 2017;64(2):275-8.

7. Josan V, Smith P, Kornberg A, Rickert C, Maixner W. Development of a pilocytic astrocytoma in a dysembryoplastic neuroepithelial tumor. Case report. J Neurosurg. 2007;106(Suppl 6):509-12.

8. Nasit JG, Shah P, Zalawadia H. Coexistent dysembryoplastic neuroepithelial tumour and pilocytic astrocytoma. Asian J Neurosurg. 2016;11(4):451.

9. Rosselló A, Plans G, Vidal-Sarró N, Fernández-Coello A, Gabarrós A. Ganglioglioma progression to combined anaplastic ganglioglioma and anaplastic pleomorphic xanthoastrocytoma. Case report and literature review. World Neurosurg. 2017;108:996.e17-25.

10. Zakrzewski K, Biernat W, Liberski PP, Polis L, Nowoslawska E. Pilocytic astrocytoma as a predominant component of a recurrent complex type DNT. Folia Neuropathol. 2009;47(3):284-8.

11. Braoudaki M, Lambrou Gl, Giannikou K, Papadodima SA, Lykoudi A Stefanaki K, et al. miR-15a and miR-24-1 as putative prognostic microRNA signatures for pediatric pilocytic astrocytomas and ependymomas. Tumour Biol. 2016;37(7):9887-97.

12. Ho CY, Bar E, Giannini C, Marchionni L, Karajannis MA, Zagzag D, et al. MicroRNA profiling in pediatric pilocytic astrocytoma reveals biologically relevant targets, including PBX3, NFIB, and METAP2. Neuro-Oncology. 2013; 15(1):69-82.

13. Kojima M, Sudo H, Kawauchi J, Takizawa S, Kondou S, Nobumasa H, et al. MicroRNA markers for the diagnosis of pancreatic and biliary-tract cancers. PLoS One. 2015;10(2):e0118220.

14. Lu J, Getz G, Miska EA, Alvarez-Saavedra E, Lamb J, Peck D, et al. MicroRNA expression profiles classify human cancers. Nature. 2005;435:834-8.
15. Vogelstein B, Papadopoulos N, Velculescu VE, Zhou S, Diaz LA Jr, Kinzler KW. Cancer genome landscapes. Science. 2013;339(6127):1546-58.

16. Thornton C, Hutter F, Hoos HH, Leyton-Brown K. Auto-WEKA: combined selection and Hyperparameter optimization of classification algorithms. In: Proceedings of the 19th ACM SIGKDD international conference on knowledge discovery and data mining. New York: ACM; 2013. p. 847-55. https://doi.org/10.1145/2487575.2487629.

17. Frank $E$, Hall $M$, Trigg L, Holmes $G$, Witten $I H$. Data mining in bioinformatics using Weka. Bioinformatics. 2004;20(15):2479-81.

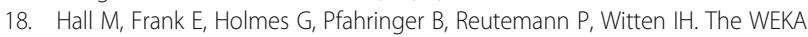
data mining software. SIGKDD Explor Newsl. 2009;11(1):10.

19. Guyon I, Weston J, Barnhill S, Vapnik VN. Gene selection for cancer classification using support vector machines. Mach Learn. 2002;46. https:// doi.org/10.1023/A:1012487302797.

20. Sticht C, De La Torre C, Parveen A, Gretz N. miRWalk: an online resource for prediction of microRNA binding sites. PLoS One. 2018;13(10):e0206239.

21. Andersen $\mathrm{CL}$, Jensen $\mathrm{JL}$, Ørntoft TF. Normalization of realtime quantitative reverse transcription-PCR data: a model-based variance estimation approach to identify genes suited for normalization, applied to bladder and colon cancer data sets. Cancer Res. 2004;64:5245-50.

22. Majumdar A, Ahmad F, Sheikh T, Bhagat R, Pathak P, Joshi SD, et al. miR217-casein kinase-2 cross talk regulates ERK activation in ganglioglioma. J Mol Med (Berl). 2017;95(11):1215-26.

23. Braoudaki M, Lambrou Gl, Papadodima SA, Stefanaki K, Prodromou N, Kanavakis E. MicroRNA expression profiles in pediatric dysembryoplastic neuroepithelial tumors. Med Oncol. 2016;33(1):5.

24. Ames HM, Yuan M, Vizcaíno MA, Yu W, Rodriguez FJ. MicroRNA profiling of low-grade glial and glioneuronal tumors shows an independent role for cluster 14q32.31 member miR-487b. Mod Pathol. 2017;30(2):204-16.

25. Prabowo AS, van Scheppingen J, lyer AM, Anink JJ, Spliet WG, van Rijen PC, et al. Differential expression and clinical significance of three inflammationrelated microRNAs in gangliogliomas. J Neuroinflammation. 2015;12:97.

26. Conti A, Romeo SG, Cama A, La Torre D, Barresi V, Pezzino G, et al. MiRNA expression profiling in human gliomas: upregulated miR-363 increases cell survival and proliferation. Tumour Biol. 2016;37(10):14035-48.

27. Iyer A, Zurolo E, Prabowo A, Fluiter K, Spliet WG, van Rijen PC, et al. MicroRNA-146a: a key regulator of astrocyte-mediated inflammatory response. PLoS One. 2012;7(9):e44789.

28. Zhou J, Wang W, Gao Z, Peng X, Chen X, Chen W, et al. MicroRNA-155 promotes glioma cell proliferation via the regulation of MXI1. PLoS One. 2013;8:e83055.

29. Yan Z, Che S, Wang J, Jiao Y, Wang C, Meng Q. miR-155 contributes to the progression of glioma by enhancing Wnt/B-catenin pathway. Tumour Biol. 2015;36(7):5323-31.

30. Schliesser MG, Claus R, Hielscher T, Grimm C, Weichenhan D, Blaes J, et al. Prognostic relevance of miRNA-155 methylation in anaplastic glioma. Oncotarget. 2016;7(50):82028-45.

31. Chen Z, Ma T, Huang C, Hu T, Li J. The pivotal role of microRNA-155 in the control of cancer. J Cell Physiol. 2014;229(5):545-50.

32. Carvalheira G, Oliveira MM, Takeno S, Lima FT, Meloni VA, Melaragno MI. $19 q 13.33 \rightarrow$ qter trisomy in a girl with intellectual impairment and seizures. Meta Gene. 2014;2:799-806.

33. Su W, Aloi MS, Garden GA. MicroRNAs mediating CNS inflammation: small regulators with powerful potential. Brain Behav Immun. 2016;52:1-8.

34. Ashhab MU, Omran A, Kong H, Gan N, He F, Peng J, et al. Expressions of tumor necrosis factor alpha and microRNA-155 in immature rat model of status epilepticus and children with mesial temporal lobe epilepsy. J Mol Neurosci. 2013;51:950-8.

35. Breuss MW, Nguyen A, Song Q, Nguyen T, Stanley V, James KN, et al. Mutations in LNPK, encoding the endoplasmic reticulum junction stabilizer Lunapark, cause a recessive neurodevelopmental syndrome. Am J Hum Genet. 2018;103(2):296-304.

36. Nagao M, Ogata T, Sawada Y, Gotoh Y. Zbtb20 promotes astrocytogenesis during neocortical development. Nat Commun. 2016;7:11102.

37. Zhang C, Cheng W, Ren X, Wang Z, Liu X, Li G, et al. Tumor purity as an underlying key factor in glioma. Clin Cancer Res. 2017;23(20):6279-91.

38. He Z, You C, Zhao D. Long non-coding RNA UCA1/miR-182/PFKFB2 axis modulates glioblastoma-associated stromal cells-mediated glycolysis and invasion of glioma cells. Biochem Biophys Res Commun. 2018;500(3):569-76.

39. Katano H, Nishikawa Y, Yamada H, Yamada K, Mase M. Differential expression of microRNAs in severely calcified carotid plaques. J Stroke Cerebrovasc Dis. 2018;27(1):108-17. 
40. Zhang T, Jing L, Li H, Ding L, Ai D, Lyu J, et al. MicroRNA-4530 promotes angiogenesis by targeting VASH1 in breast carcinoma cells. Oncol Lett. 2017;14(1):111-8.

41. Xun M, Ma CF, Du QL, Ji YH, Xu JR. Differential expression of miRNAs in enterovirus 71-infected cells. Virol J. 2015;12:56.

42. Hasegawa T, Glavich GJ, Pahuski M, Short A, Semmes OJ, Yang L, et al. Characterization and evidence of the miR-888 cluster as a novel cancer network in prostate. Mol Cancer Res. 2018;16(4):669-81.

43. Yao S, Hu M, Hao T, Li W, Xue X, Xue M, et al. MiRNA-891a-5p mediates HIV1 tat and KSHV Orf-K1 synergistic induction of angiogenesis by activating NF-kB signaling. Nucleic Acids Res. 2015:43(19):9362-78.

44. Li M, Qian Z, Ma X, Lin X, You Y, Li Y, et al. MiR-628-5p decreases the tumorigenicity of epithelial ovarian cancer cells by targeting at FGFR2. Biochem Biophys Res Commun. 2018;495(2):2085-91.

45. Megiorni F, Colaiacovo M, Cialfi S, McDowell HP, Guffanti A, Camero S, et al. A sketch of known and novel MYCN-associated miRNA networks in neuroblastoma. Oncol Rep. 2017;38(1):3-20.

46. Moretti F, D'Antona P, Finardi E, Barbetta M, Dominioni L, Poli A, et al. Systematic review and critique of circulating miRNAs as biomarkers of stage I-II non-small cell lung cancer. Oncotarget. 2017;8(55):94980-96.

47. Wang Y, Zhao H, Gao X, Wei F, Zhang X, Su Y, et al. Identification of a threemiRNA signature as a blood-borne diagnostic marker for early diagnosis of lung adenocarcinoma. Oncotarget. 2016;7(18):26070-86.

48. Díez-Planelles C, Sánchez-Lozano P, Crespo MC, Gil-Zamorano J, Ribacoba R, González N, et al. Circulating microRNAs in Huntington's disease: emerging mediators in metabolic impairment. Pharmacol Res. 2016;108:102-10.

49. Tian Y, Hao S, Ye M, Zhang A, Nan Y, Wang G, et al. MicroRNAs let-7b/i suppress human glioma cell invasion and migration by targeting IKBKE directly. Biochem Biophys Res Commun. 2015;458(2):307-12.

50. Song H, Zhang Y, Liu N, Zhang D, Wan C, Zhao S, et al. Let-7b inhibits the malignant behavior of glioma cells and glioma stem-like cells via downregulation of E2F2. J Physiol Biochem. 2016;72(4):733-44.

51. Weingart MF, Roth JJ, Hutt-Cabezas M, Busse TM, Kaur H, Price A, et al. Disrupting LIN28 in atypical teratoid rhabdoid tumors reveals the importance of the mitogen activated protein kinase pathway as a therapeutic target. Oncotarget. 2015;6(5):3165-77.

52. Byrne JA, Frost $\mathrm{S}$, Chen Y, Bright RK. Tumor protein D52 (TPD52) and cancer-oncogene understudy or understudied oncogene? Tumour Biol. 2014;35(8):7369-82.

53. Donzelli S, Mori F, Bellissimo T, Sacconi A, Casini B, Frixa T, et al. Epigenetic silencing of miR-145-5p contributes to brain metastasis. Oncotarget. 2015; 6(34):35183-201.

54. McDermott N, Meunier A, Wong S, Buchete V, Marignol L. Profiling of a panel of radioresistant prostate cancer cells identifies deregulation of key miRNAs. Clin Transl Radiat Oncol. 2017;2:63-8.

55. Wagenseller AG, Shada A, D'Auria KM, Murphy C, Sun D, Molhoek KR, et al. MicroRNAs induced in melanoma treated with combination targeted therapy of Temsirolimus and bevacizumab. J Transl Med. 2013:11:218.

56. Bongaarts A, Prabowo AS, Arena A, Anink JJ, Reinten RJ, Jansen FE, et al. MicroRNA519d and microRNA4758 can identify gangliogliomas from dysembryoplastic neuroepithelial tumours and astrocytomas. Oncotarget. 2018;9(46):28103-15.

\section{Publisher's Note}

Springer Nature remains neutral with regard to jurisdictional claims in published maps and institutional affiliations.

Ready to submit your research? Choose BMC and benefit from:

- fast, convenient online submission

- thorough peer review by experienced researchers in your field

- rapid publication on acceptance

- support for research data, including large and complex data types

- gold Open Access which fosters wider collaboration and increased citations

- maximum visibility for your research: over $100 \mathrm{M}$ website views per year

At BMC, research is always in progress.

Learn more biomedcentral.com/submissions 